\title{
An Updated Review on Covid-19 with Special Reference to Structural Elucidation and Functional Properties
}

\author{
Tugba Taskin Tok ${ }^{1,2 *}$ and Sivakumar J T Gowder ${ }^{3,4 *}$ \\ ${ }^{1}$ Department of Chemistry -Faculty of Arts and Science, Gaziantep University, Gaziantep, Turkey \\ ${ }^{2}$ Department of Bioinformatics and Computational Biology - Institute of Health Sciences, Gaziantep University, Gaziantep, \\ Turkey
}

${ }^{3}$ Bionanotechnology Research Group, Ton Duc Thang University, Ho Chi Minh City, Vietnam

${ }^{4}$ Faculty of Pharmacy, Ton Duc Thang University, Ho Chi Minh City, Vietnam

*Corresponding author: Tugba Taskin Tok, Department of Chemistry, Faculty of Arts and Science, Gaziantep University, Gaziantep, Turkey; Department of Bioinformatics and Computational Biology, Institute of Health Sciences, Gaziantep University, Gaziantep, Turkey

Sivakumar J T Gowder, Ton Duc Thang University, Ho Chi Minh City, Vietnam

\section{ARTICLE INFO}

Received: 彗 October 16, 2020

Published: 㐩 November 04, 2020

Citation: Tugba Taskin Tok, Sivakumar J T Gowder. An Updated Review on Covid-19 with Special Reference to Structural Elucidation and Functional Properties. Biomed J Sci \& Tech Res 31(4)-2020. BJSTR. MS.ID.005131.

\begin{abstract}
COVID-19, the RNA virus is considered to be the most dangerous microbe in the history of the mankind. Both the developed and developing countries are struggling for about three fourth of this whole year-2020, to prevent this disease but in vain. The fatality ratio and widespread of the diseases caused by COVID-19 are dangerous to global human population. Coronaviruses are exhibiting the crown-like spikes on their body surface. A coronaviral genome encodes four major structural proteins: spike $(S)$ protein, nucleocapsid $(\mathrm{N})$ protein, membrane $(\mathrm{M})$ protein, and the envelope (E) protein. All these proteins are necessary to grow a complete viral particle. Each protein has its own structural and functional role with reference to viral growth and infection. After entering human body, the viral particles can interact immediately with angiotensin-converting enzyme 2 to enter target cells. Though the outbreak of COVID-19 in China is unknown but the wide spread of this infection reveals that it can spread from animals to human and human to human and also through air. In this article, we have discussed about the structural aspects, functional properties, preventive measures of COVID-19 and also the updated information on the development of medicines and vaccines for the disease caused by COVID-19.
\end{abstract}

\section{Introduction}

Coronaviruses (CoVs) are enveloped, single-stranded and wide families of RNA viruses and have four subgroups: alpha, beta, gamma, and delta. The title "Coronavirus" comes from the Latin word where corona means 'crown' or 'halo;' because of the crownlike projections on the surface of the virus. The viruses cause ill from the cold (common human coronaviruses having types $\mathrm{HCoV}$ HKU1, HCoV-NL63, HCoV-229E and HCoV-OC43) to more serious diseases like SARS-CoV (the beta coronavirus that causes severe acute respiratory syndrome, or SARS) in 2002, MERS-CoV (the beta coronavirus that causes Middle East respiratory syndrome, or

MERS) in 2012 and SARS-CoV-2 (the novel coronavirus that causes Coronavirus Disease 2019, or COVID-19) in 2019 [1] (Figure 1). The scientists have reported that coronavirus can infect from animal species to human beings. It means that the loud fatality ratio and widespread of these novel emerging coronaviruses are a violent danger to global human population. Common human coronaviruses (NL63, HKU1, 229E, and OC43) provoke infection in humans. They induce common cold through the respiratory tract. There is no remedy for the virus induced diseases. SARS-CoV is a beta coronavirus that induces severe acute respiratory syndrome, SARS- 
$\mathrm{CoV}$, was transmitted from civet cats to humans. This syndrome is the severe type of pneumonia. The outbreak of SARS exit in 2002 and was liable for an epidemical that radiate to five continents with a fatality ratio of $10 \%$ before being in 2003 (with additional cases reported in 2004) [1].

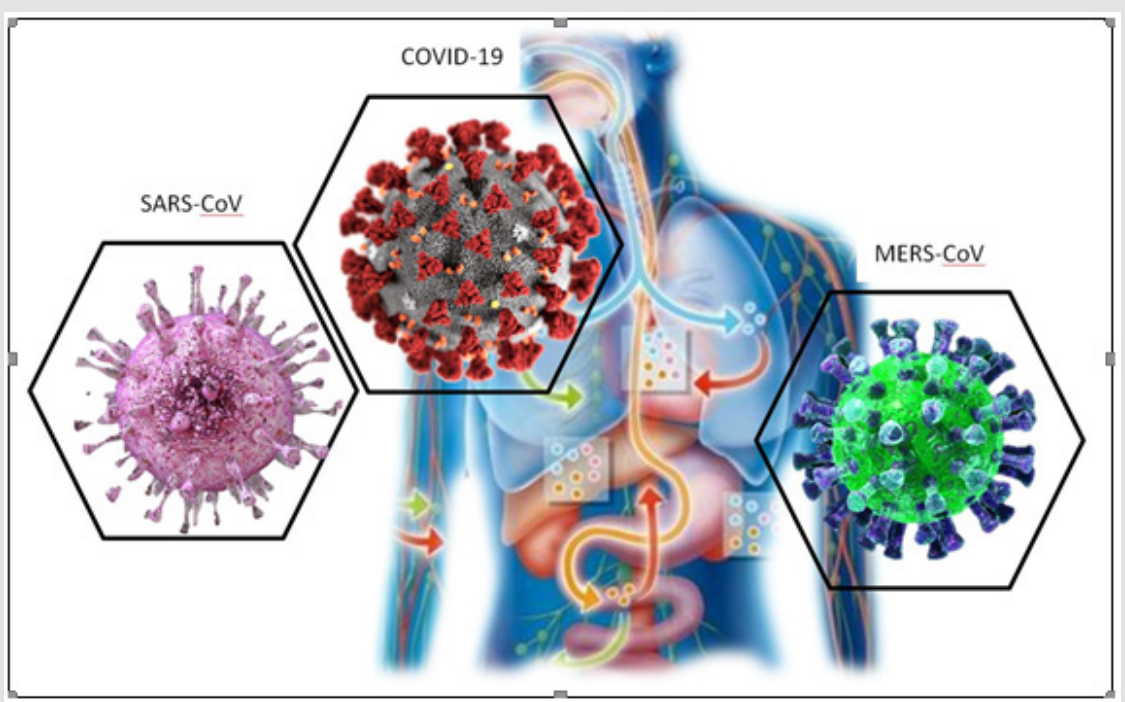

Figure 1: Diagram of severe three type models of human coronavirus.

MERS-CoV produces Middle Eastern Respiratory Syndrome (MERS) that is a severe disease. This virus was transmitted on from dromedary camels to humans and the outbreak of this virus was noticed first in South Korea in the year 2015. The newest coronavirus, COVID-19 was first noticed in Wuhan city, Hubei Province in China. Chinese scientists recognized the new coronavirus, which were found in hundreds of confirmed cases in China, having cases outside Wuhan City, with additional cases being defined in a growing number of countries internationally. The first case in the United States was advertised on January 21, 2020. The novel coronavirus is a new type of virus that there are ongoing examinations are on the way [2]. Mutual indications of infection contain respiratory symptoms, fever, and cough, shortness of breath and breathing difficulties. In more severe cases, infection can procure pneumonia, severe acute respiratory syndrome, kidney failure and even death. The deficiency of powerful drug cure and united high morbidity and mortality ratio of these two CoVs and their potency to procure epidemics highlight the need for new drug discovery to use $\mathrm{CoV}$ infections. Canonical advices to prohibit infection spread include regular hand washing and covering mouth and nose when coughing and sneezing. You refrain close contact with anyone who indicates symptoms of respiratory illness such as coughing and sneezing [3].

\section{Structure and Properties of Coronavirus}

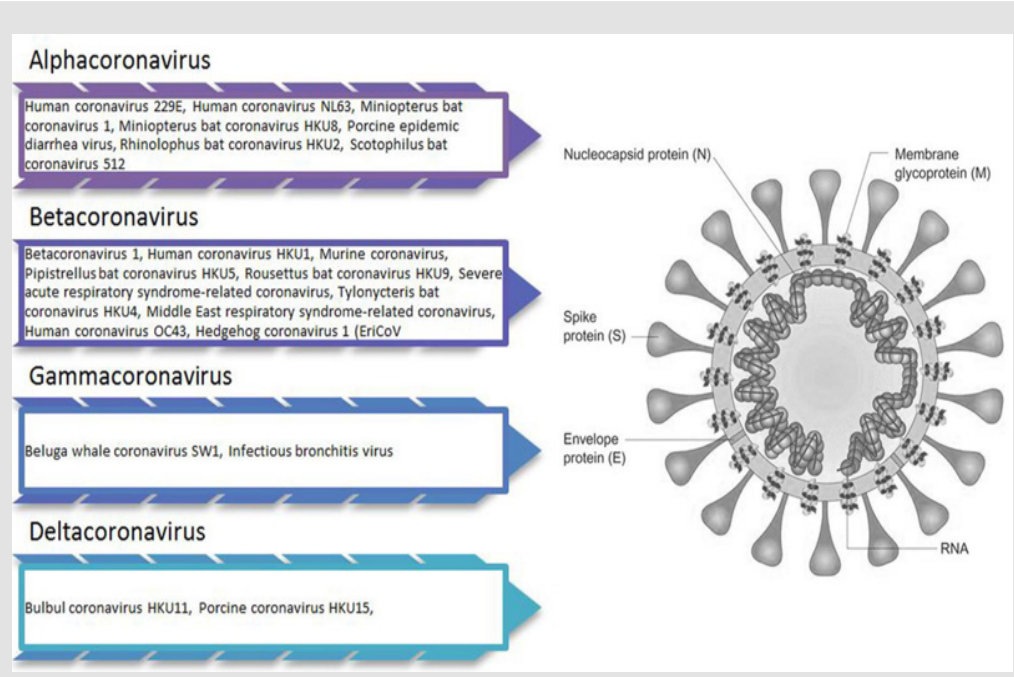

Figure 2: Organization of CoV species [3] (Coronavirus From Wikipedia, 2020). 
Coronaviruses are exhibiting the crown-like spikes on their body surface. They are part of the Coronavirinae family of the Nidovirales group. There are four genera of coronaviruses - alpha, beta, gamma, and delta (Figure 2). A coronaviral genome encodes four major structural proteins. These are spike $(\mathrm{S})$ protein, nucleocapsid $(\mathrm{N})$ protein, membrane $(\mathrm{M})$ protein, and the envelope (E) protein and all of them are necessary particles to grow a structurally exact viral [4]. However, it has recently been reported that some CoVs do not need the complete structural protein community to form a complete, infectious virion [5 -7]. Individually, each protein plays a role primarily in the structure of the virus particle, but they also play a role in other aspects of the replication cycle. The spike (S) glycoprotein of the coronavirus is a class I viral fusion protein on the outer envelope of the virion that moves a crucial role in viral infection by recognizing host cell receptors and mediating fusion of the viral and cellular membranes [8 -10]. It is synthesized as a precursor protein comprising $\sim 1,300$ amino acids. There are two functional subunits of S: subunit-S1 and subunit-S2. S1 subunit favors binding to the host cell receptor and S2 subunit is responsible for the fusion of the viral and cellular membranes.

Coronavirus $\mathrm{S}$ glycoprotein features two diverse protease cleavage spaces. The S1/S2 cleavage space of the SARS-CoV S glycoprotein is placed after residue 667 of the precursor protein, but the S2' cleavage space of the SARSCoV S glycoprotein is on the
S2 subunit and is 130 amino acids from the $\mathrm{N}$ terminus of the S2 subunit. The S1/ S2 cleavage site is involved in the perfusion of the S1-S2 trimer spike. The S2 cleavage site is involved in the perfusion of SARS-CoV S glycoprotein [11-13]. Constructional biology works, especially recent cryo-electron microscopy (cryo-EM) works have evolved our mentality on the act of coronavirus $S$ glycoprotein in virus inlet. $S$ glycoprotein structures have been noticed from the

alpha coronavirus genus (HCoV-NL63), beta coronavirus genus (mouse hepatitis virus (MHV), HKU1, SARS-CoV and MERS-CoV), gamma coronavirus genus (IBV), and delta coronavirus genus (PdCoV) [14-22]. Here, we report S glycoprotein genomes and constructions of the SARS-CoV, MERS-CoV and BatCoV observed by cryo-EM. Based on these structures, COVID-19 structure is being tried to define in the same approaches (Figure 3). Further, SARS$\mathrm{CoV}$ and several SARS-related coronaviruses (SARS-CoV) interact immediately with angiotensin-converting enzyme 2 (ACE2) to enter into target cells [23]. Recent studies show that ACE2 can enter the cells, accompanied by SARS-CoV-2 S, and establish this protein as a functional receptor for this emerging coronavirus. Strict binding to hACE2 could partly elucidate the effective transmission of SARS CoV- 2 in humans, as was the case for SARS-CoV. At the same time, cryo-electron microscopic structure of the SARS-CoV-2 S ectodomain trimer shows that SARS-CoV S and MERS-CoV S follow a similar course in the transition to target cells.

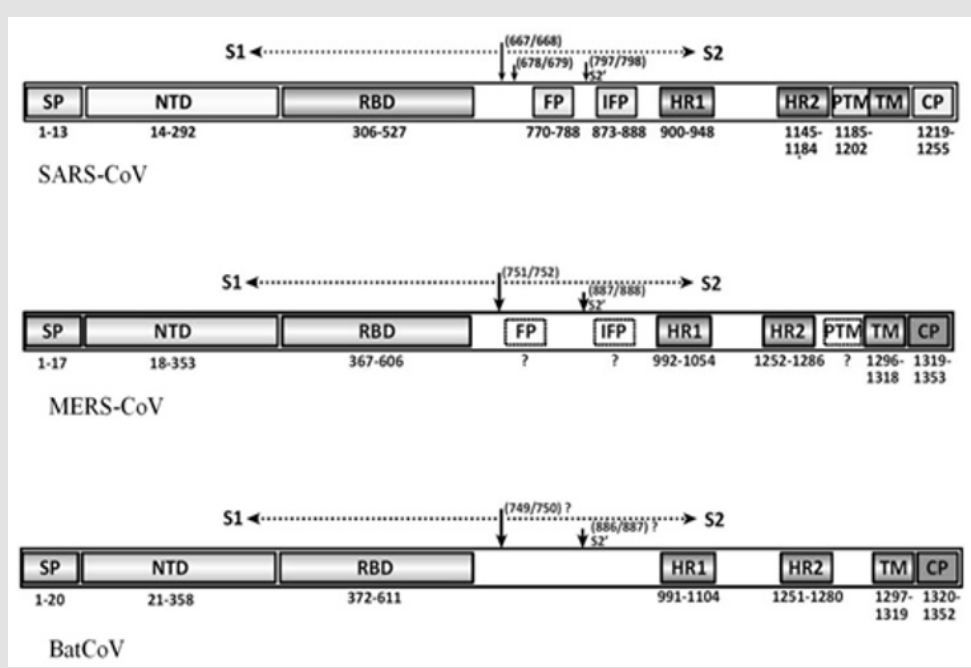

Figure 3: Schematic representation of the SARS-CoV, MERS-CoV and BatCoV spike proteins (S). The individual components of S including receptor-binding domain (RBD), fusion peptide (FP), internal fusion peptide (IFP), heptad repeat 1/2 (HR1/2), and pre transmembrane domain (PTM) $[13,27,35]$ - or are based on bioinformatics analyses, for example, N-terminal domain (NTD), are marked with the boundary-residue numbers listed below. The S1/S2 cleavage sites and the S2'-recognition site are highlighted. Other abbreviations: SP, signal peptide; TM, trans membrane domain; and CP, cytoplasmic domain.

\section{Coronavirus Outbreak - China}

COVID-19 was first identified (Figure 4), among employees or customers of a large seafood and animal market in Wuhan city, Hubei province of China. However, it has been understood that the virus can sperad from person to person through little droplets from the nose or mouth when the disease is diagnosed in people not related to this market. It was also noticed that there are a limited number of cases in Thailand, Japan, Taiwan, South Korea, the USA and Europe. Although initially, it is believed to be transmitted from animals to human, there are now many examples of COVID-19 transmission from person to person. Reports also show that coronaviruses were noticed in Chinese wild bats at different time periods. When these 
wild animals are caught and killed for food, there will be a huge rate of infection. Other wild animals such as snakes, hedgehogs, rhizomes and pangolins are coronavirus carriers based on scientific findings. It is alarmed that it would be safe not to eat food made from these animals. The WHO reported many COVID -19 cases in different geographical regions of the world. Based on these results, they reported contamination from person to person; however, the vast majority of cases are said to be related to Chinese citizens travel history to Wuhan City and also other cities in China or in the world. While deaths are clearly important to those involved directly and indirectly, the novelty and unknown trajectory of the outbreak creates it newsworthy. Given that, the magnitude of more deaths is confined to common flu at large, scientists are perhaps concerned with revising and studying the flu in detail.

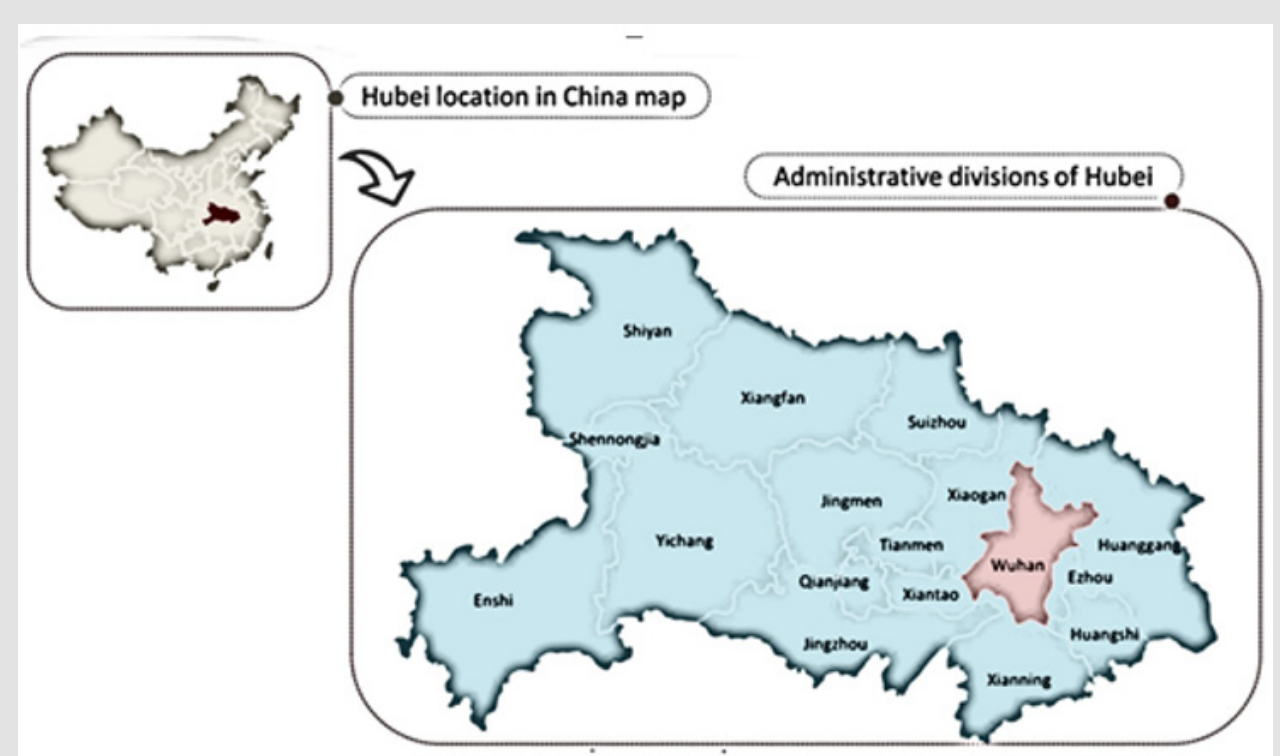

Figure 4: Wuhan city, Hubei province of China.

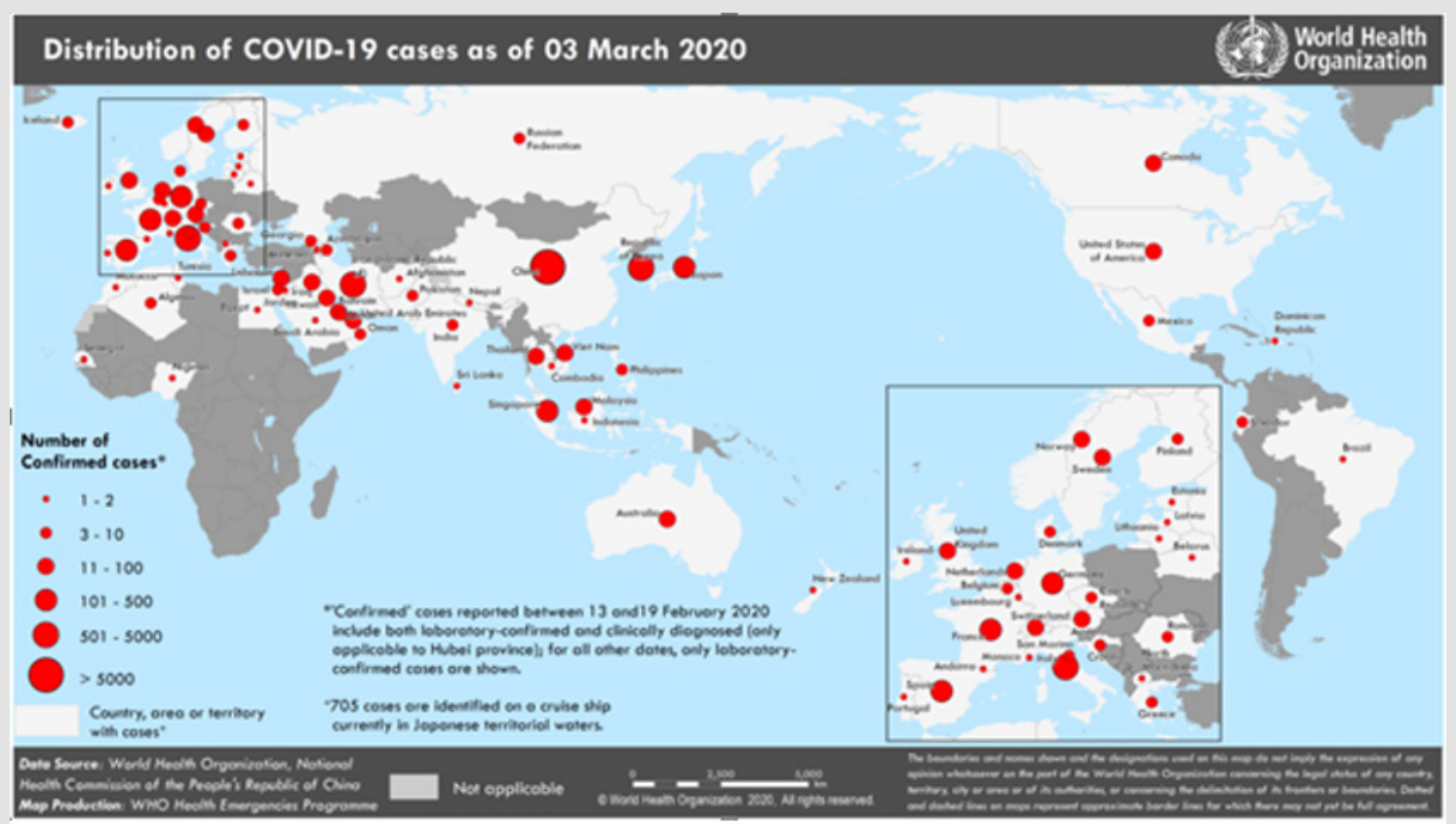

Figure 5: Countries / territories or areas - status of COVID-19: March -2020 [25].

These virus-borne droplets reach near their mouths and noses and settle in their lungs when people breathe. All other routes of transmission of the coronavirus have not yet been fully described.
However, the surface and contact can transmit it. The incubation period of the coronavirus (COVID-19) (that is, the time between exposure to the virus and the start of symptoms) is now predicted 
from two to 14 days. Others should think of it as the most infectious stage of the respiratory virus when people are unhealthy. However, in some coronavirus cases, the spread has been detected from an infected patient who has not yet shown any signs of disease. This confirms whether early detection of COVID-19 infections will be difficult. Therefore, the risk rate it creates will be more. In summary, human can be infected COVID-19 easily from another person who has the virus. This disease is spread when a person with COVID-19 either coughs or exhales. Other possibility, person can be infected it by touching these objects and/or surfaces; if person breathes in droplets from a person with COVID-19. Because of these states, a person should stay more than one meter away from infected people. In March-2020, based on various reliable sources, there were 90 870 reported cases and 166 confirmed deaths from COVID-19 [24] (Figure 5).

The reason for this viral outbreak and infection is unknown. The information is largely limited to what is known about similar corona viruses. The virus mostly seen in animals can rarely cause infection in humans. However, when it is infected, it transmits, that it is easily transmitted to other people, such as the flu, from being easily infected. In particular, novel coronavirus has features such as common cold, severe acute respiratory syndrome (SARS) and Middle East respiratory syndrome (MERS), which are part of the coronavirus family. Since it was first described in Saudi Arabia in 2012, 858 of nearly 2494 cases of people reportedly infected with MERS have died. In the SARS outbreak, 8098 identified cases and 774 died. The current COVID-19 pandemic is incomparable than its predecessors. According to the date of 09 March 2020, it is stated that the COVID-19 outbreak caused 109,343 identified cases and 3,809 deaths [25]. As of 15 - October 2020, the confirmed cases of COVID-19: 38,202,956 and deaths: 1,087,069 (global data). The data of the COVID-19 infection rate in WHO regions are the following (confirmed cases): Americas - 18,185,839; South-East Asia- 8,204,366; Europe- 7,219,501; Eastern Mediterranean 2,684,378; Africa - 1,240,683; Western Pacific- 667,448 [26]. When we compare the data of March with that of the data of October, we can notice that there been an increase of 350 folds infected cases and an increase of 285 folds death cases. When we start to do review research on COVID-19 to write this article (March, 09) and at the time of updating this article for submission (Oct, 15), in between a long gap, we can notice rapid in increase of infection rate in addition to the death toll, across the world.

\section{Health Education: Coronavirus Prevention}

Coronavirus has been confirmed to be transmitted from person to person by droplets. When infected, breathing, coughing, sneezing, speaking, and touching, infected droplets spread to people with a diameter of about one to two meters. When the virus leaves the patient, it will be difficult for them to survive in the air. This disease has five clear symptoms: fever, dry cough, shortness of breath, fatigue and respiratory distress (Figure 6). The most evident symptom is fever, whose body temperature is greater than 37.3 Celsius degrees. We should protect ourselves and prevent the spread of this disease. We can clean our hands regularly and by rubbing an alcohol-based hand sanitizer or wash them with soap and water. Since we wash our hands with soap and water or use alcohol-based hand scrubs, they kill viruses that may be in our hands. We can leave a distance of at least 1 meter ( 3 feet) between yourself and anyone who coughs or sneezes. When someone coughs or sneezes, it sprays little drops of liquid that can contain viruses from their nose or mouth. If we are very close, we can inhale the droplets that contain COVID-19 virus. You should refrain contact the mouth, eyes, and nose. Because your hands handle a great deal of surfaces and can meet viruses. When dirty, your hands can get over the virus to your eyes, nose, or mouth. From there, the virus can spread our our body and infect us.

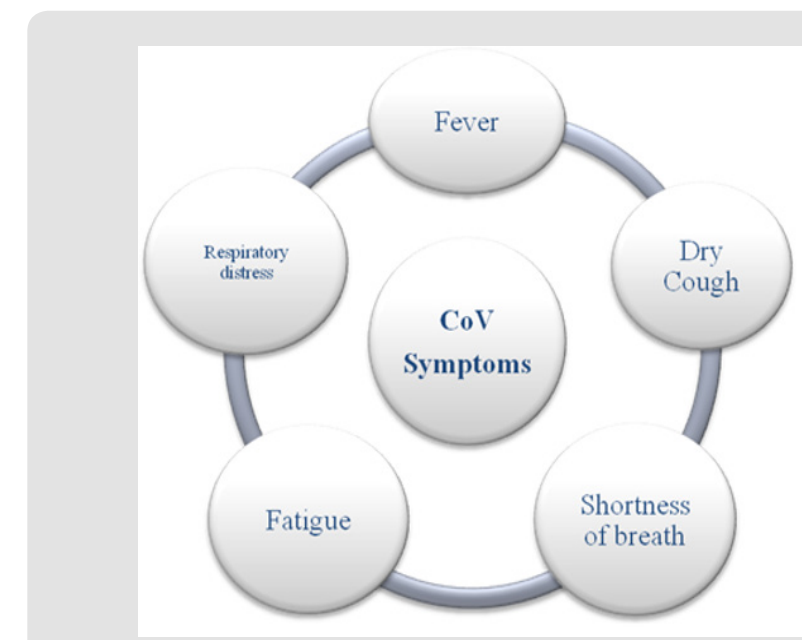

Figure 6: Coronavirus symptoms.

We should be sure to apply fine respiratory hygienic. This implies that skin in our mouth and nose with a folded elbow or tissue when you cough or sneeze. Then immediately discard the used tissue or napkin, because droplets radiate the virus. By applying good respiratory hygiene, we keep people around us from viruses such as colds, flu and COVID-19. If we feel ill, we need to stay at home. If we have a fever, cough, and trouble breathing, we need to call medical advice and call in advance. Observe the instructions of our regional health center. Because public and regional authorities will have the most up-to-date knowledge about the status in our region. Searching in the future will allow oour healthcare professional to fast guide us to the correct healthcare professional. This will also keep us to prohibit spreading of viruses and other infections. One should hold the latest COVID-19 hotspots (cities or local regions where COVID-19 is widely splay) up to date. If possible, we should refrain traveling to places, particularly if we are an elderly person or have diabetes, heart or lung disease. Elderly people and people with health complications have a higher risk of COVID-19 infections irrespective of geographical regions. 
Besides, we should monitor the people who have visited COVID-19 regions (last 14 days) or recently traveled abroad. We need to apply the protection measures specified for everyone engaged in travel. Infected people should rest at home until they recover, even with slight signs such as headaches, low-grade fever (37.3 C or higher), and mild nasal discharge. One should wear a mask for every occasion. After travel, if we have flue, cough or breathing problems, we need to call the primary health care centers or physicians near to our area for medical advice in advance. Because, making a call ahead of time will let our healthcare provider to fast guide us to the correct healthcare center. This will also assist in prohibiting the probable spread of COVID-19 and other viruses.

\section{Medicines / Vaccines}

To date, there is no special remedy for COVID -19. Remedy is determined by symptoms. Some western, traditional or home remedies can provide comfort and relieve the symptoms of COVID-19. Therefore, there is no evidence that a particular drug can prevent or treat the disease. WHO has not so far recommended any kind of medicine or antibiotics to prevent COVID-19. However, there are several ongoing clinical trials involving both western and traditional medicines. Anyhow, WHO is keen on providing updated information for the people about research and clinical findings concerned to COVID-19. For example, when a person has a high fever with breathing problems, oxygen therapy is given. Such supportive treatments can be quite effective for infected patients. In addition, a vaccine and a specific antiviral drug are not yet available to prevent or treat COVID-19. However, there are practices to alleviate the symptoms of those affected until now. People with complicated disease should be hospitalized. Probable vaccines and some private medicine therapies are still under examination. It is then provided by clinical testing to timely publish epidemic information to check the origin of infection, early detection, reporting, isolation, supportive therapies, and to avoid unnecessary panic. Many countries are undergoing extensive research to develop coronavirus vaccines.

Further, the Wall Street Journal reported that several drug manufacturers are competing to develop vaccines that can protect against the new Chinese respiratory virus, (COVID-19). Many health based industries - Moderna Inc., Inovio Pharmaceuticals Inc. and Novavax Inc. plans to develop a vaccine against the newly identified viral strain. Researchers at Queensland University in Australia are also trying to develop a vaccine against these strains [27]. Recently, Fierce Biotech reported that both JNJ and Gilead jumped into the accelerated Coronavirus Vaccine race. WHO also coordinates vaccine and drug development efforts to prevent and treat COVID-19. Besides these, researchers are testing countermeasures for 2019-nCoV using SARS-CoV and MERS-CoV as prototypes [28]. Effective antivirals - remdesivir (RNA polymerase inhibitor); lopinavir and interferon beta which have been used for MERS-CoV in animal models and are being evaluated for 2019.
$\mathrm{nCoV}$. Vaccines (and antibody), with nucleic acid vaccine platform approaches used for SARS-CoV or MERS-CoV, are being followed at the National Institute of Allergy and Infectious Diseases Vaccine Research Center. The most potent paths to keep yourself and others against COVID-19 are to clean your hands frequently, cloak your cough with elbow or tissue curl, and provide a interval of at least 1 meter (3 feet) from people who cough or sneeze.

One of the authors of this article, in his editorials has revealed that advance scientific methods in addition to traditional measures can prevent COVID-19 infection at certain extent; South Indian traditional food (rasam) will be helpful to control COVID-19; eating meat and meat products are unsafe in this pandemic and the role of health education in preventing the COVID-19 infection. There is an arithmetic model to evaluate the rate of COVID-19 infection [2933].

\section{Conclusion}

The occurrence of two betaCoV-related outbreaks over the previous decade has stimulated COVID-19 investigative, focusing on the cross-species transmission mechanisms of these viruses. In particular, the dense academic struggle to identify SARS and SARS$\mathrm{CoV}$ provided an inimitable status study in viral evolution and zoonotic transmission. The example of SARS-CoV underlines the need for a type of bridge that can guide virus development in some cases, allowing direct emergence of a variant that is in direct contact with people and can be efficiently transmitted between humans. It highlights viral strategies that allow quick conformation to novel kinds and indicates that mild pathogenic viruses may not stay that way with variances in human and animal populations, that raise viral variation or interspecific contact density. Experimentation with SARS-CoV has represented the significance of fieldwork that definitive and describes viruses and host agents in feral and domestic animals. Forward study on these guidelines can aid to predict and prevent the latter SARS. Moreover, CoV S protein is an important element to be investigated in this time point of view. Much progression has been accomplished so far on the properties of $S$, the interplay of $S$ with receptors and the preparation of $S$ by host proteases. In a nutshell, this study is focused on our mentality of coronaviral S proteins to demonstrate the base for cross-species transmission of SARS-CoV, MERS-CoV and beyond this information should assist to prohibit or estimate forward transmission cases.

\section{References}

1. ChenY, Liu Q Guo D (2020) Emerging coronaviruses: Genome structure, replication, and pathogenesis. J Med Virol 92(4): 418-423.

2. Christian CG, Georg S (2020) Comparative model of novel coronavirus 2019-nCoV protease Mpro.

3. (2020) Coronavirus From Wikipedia. Taxonomy from From Wikipedia, the free encyclopedia.

4. Tok T, Tatar G (2017) Structures and Functions of Coronavirus Proteins: Molecular Modeling of Viral Nucleoprotein. International Journal of Virology \& Infectious Diseases 2(1): 001-007. 
5. De Diego ML, Alvarez E, Almazan F, Rejas MT, Lamirande E, et al. (2007) A severe acute respiratory syndrome coronavirus that lacks the E gene is attenuated in vitro and in vivo. J Virol 81(4): 1701-1713.

6. Kuo L, Masters PS (2003) The Small Envelope Protein E Is Not Essential for Murine Coronavirus Replication. J Virol 77(8): 4597-4608.

7. Mortola E, Roy P (2004) Efficient assembly and release of SARS coronavirus-like particles by a heterologous expression system. FEBS Lett 576(1-2): 174-178.

8. Fan Y, ZhaoK, Shi ZL, Zhou P (2019) Bat Coronaviruses in China. Viruses 11(3): 210.

9. Siu YL, Teoh KT, Lo J, Chan CM, Kien F, et al. (2008) The M, E, and N Structural Proteins of the Severe Acute Respiratory Syndrome Coronavirus Are Required for Efficient Assembly, Trafficking, and Release of Virus-Like Particles. J Virol 82(22): 11318-11330.

10. Song HC, Seo MY, Stadler K, Yoo BJ, Choo QL, et al. (2004) Synthesis and characterization of a native, oligomeric form of recombinant severe acute respiratory syndrome coronavirus spike glycoprotein. J Virol 78(19): 10328-10335.

11. Belouzard S, Chu VC, Whittaker GR (2009) Activation of the SARS coronavirus spike protein via sequential proteolytic cleavage at two distinct sites. JPNAS 106(14): 5871-5876.

12. Millet JK, Whittaker GR (2015) Host cell proteases: Critical determinants of coronavirus tropism and pathogenesis. Virus Research 202: 120-134.

13. Pallesen J, Wang N, Corbett KS, Wrapp D, Kirchdoerfer RN, et al. (2017) Immunogenicity and structures of a rationally designed prefusion MERS-CoV spike antigen. JPNAS 114(35): E7348-E7357.

14. Gui M, Song W, Zhou H, Xu J, Chen S, et al. (2016) Cryo-electron microscopy structures of the SARS-CoV spike glycoprotein reveal a prerequisite conformational state for receptor binding. Cell Res 27(1) 119-129.

15. Kirchdoerfer RN, Cottrell CA, Wang N, Pallesen J, Yassine HM, et al. (2016) Pre-fusion structure of a human coronavirus spike protein Nature 531(7592): 118-121.

16. Martin S (2020). Homology Models of Coronavirus 2019-nCoV 3CLpro Protease, Chem Rxiv.

17. Shang J, Zheng Y, Yang Y, Liu C, Geng Q et al. (2018) Cryo-EM structure of infectious bronchitis coronavirus spike protein reveals structural and functional evolution of coronavirus spike proteins. PLoS Pathog 14(4): e1007009.

18. Shang J, Zheng Y, Yang Y, Liu C, Geng Q et al. (2018) Cryo-Electron Microscopy Structure of Porcine Deltacoronavirus Spike Protein in the Prefusion State. J Virol 92(4): e01556-01517.

\section{ISSN: 2574-1241}

\section{DOI: 10.26717/BJSTR.2020.31.005131}

Tugba Taskin T, Sivakumar J T Gowder. Biomed J Sci \& Tech Res

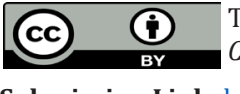

This work is licensed under Creative

Commons Attribution 4.0 License

Submission Link: https://biomedres.us/submit-manuscript.php
19. Walls AC, Tortorici MA, Bosch BJ, Frenz B, Rottier PJM, et al. (2016) Cryo-electron microscopy structure of a coronavirus spike glycoprotein trimer. Nature 531(7592): 114-117.

20. Walls AC, Tortorici MA, Frenz B, Snijder J, Li W, et al. (2016) Glycan shield and epitope masking of a coronavirus spike protein observed by cryoelectron microscopy. Nat Struct Mol Biol 23(10): 899-905.

21. Xiong X, Tortorici MA, Snijder J, Yoshioka C, Walls AC, et al (2018) Glycan Shield and Fusion Activation of a Delta coronavirus Spike Glycoprotein Fine-Tuned for Enteric Infections. J Virol 92(4): e01628-01617.

22. Yuan Y, Cao D, Zhang Y, Ma J, Qi J, et al. (2017) Cryo-EM structures of MERS-CoV and SARS-CoV spike glycoproteins reveal the dynamic receptor binding domains. Nat Commu 8: 15092.

23. Yang XL, Hu B, Wang B, Wang MN, Zhang Q, et al. (2016) Isolation and Characterization of a Novel Bat Coronavirus Closely Related to the Direct Progenitor of Severe Acute Respiratory Syndrome Coronavirus. J Virol 90(6): 3253-3256.

24. (2020a) World Health Organization, W. (Producer). (2020a) Coronavirus disease (COVID-2019) situation reports, USA.

25. (2020b) Novel Coronavirus (COVID-19) Situation. World Health Organization, USA.

26. (2019) WHO Coronavirus Disease (COVID-19) Dashboard, WHO, USA.

27. Lowe D (2020) Coronavirus., Science Translational medicine.

28. Paules CI, Marston HD, Fauci AS (2020) Coronavirus Infections-More Than Just the Common Cold. JAMA.

29. Gowder SJT (2020) Coronavirus Aftermath: An Ideal Method to Educate People. J Community Med Health Care Sys 2020 1(1): 003.

30. Gowder SJT (2020) Tamil Nadu Traditional Food - A Remedy for Coronovirus (Neuropathogen) and other Infections. Acta Scien Neurol 3(3): 01-02.

31. Gowder SJT (2020) PPSS (Philosophical, Psychological, Sociological and Scientific) Method of Preventing Coronavirus. UJEIRD 25-27.

32. Gowder SJT (2020) Is it Safe to Eat Meat or Meat Products in this Covid 19 Pandemic 1(1): 1002.

33. Gowder SJT (2020) PPSS (Philosophical, Psychological, Sociological and Scientific) Method: An Arithmetic Model to Evaluate the Potential of Covid-19 Infection. Vaccines Vacccin 5(1): 000140.

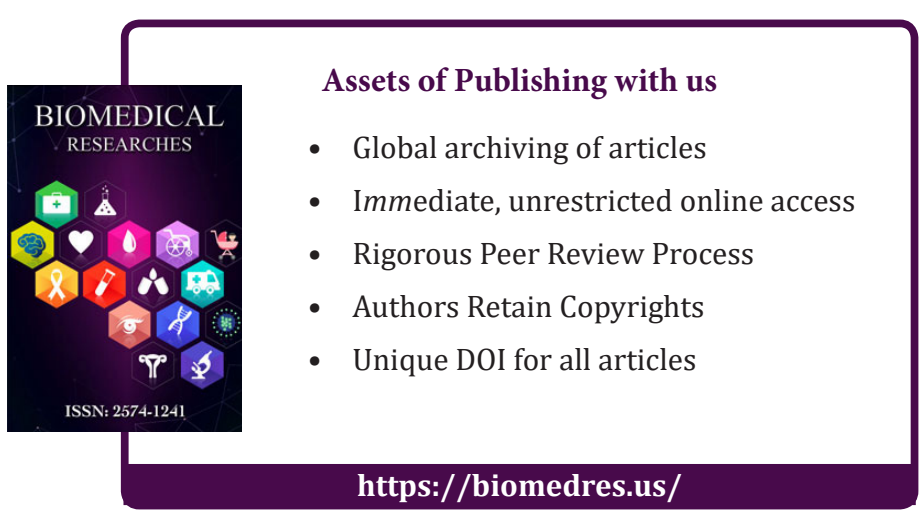

